\title{
Percutaneous pulmonary polyurethane valved stent implantation
}

\author{
Anja Metzner, PhD, ${ }^{\mathrm{a}}$ Kenji Iino, $\mathrm{MD},{ }^{\mathrm{a}}$ Ulrich Steinseifer, $\mathrm{PhD},{ }^{\mathrm{b}}$ Anselm Uebing, $\mathrm{MD},{ }^{\mathrm{c}}$ \\ Wiebke de Buhr, VMD, ${ }^{\mathrm{a}}$ Jochen Cremer, $\mathrm{MD}, \mathrm{PhD},{ }^{\mathrm{a}}$ and Georg Lutter, $\mathrm{MD}, \mathrm{PhD}^{\mathrm{a}}$
}

Objectives: Transfemoral application of pulmonary heart valves has been studied for the past 10 years. Nevertheless, size restriction of percutaneous heart valved stents is still imminent.

\begin{abstract}
Methods: In this study we implanted percutaneously a novel, low-profile polyurethane valved stent. Percutaneous implantation in pulmonary position was evaluated in 7 sheep. The new valved stent fits into a $14 \mathrm{~F}$ delivery device. The self-expanding nitinol stent was produced by using a dip-coating technique, and a modified commercially available endovascular stent graft system served as a delivery device. The valved stents were deployed directly over the native pulmonary valve under fluoroscopic control. Transthoracic echocardiography was performed after 4 weeks. At the time of explantation, the animals were reanalyzed and killed. Angiography was performed at implantation and at the end of the study. Explanted constructs were analyzed macroscopically and microscopically.
\end{abstract}

Results: Angiography and echocardiography in all animals demonstrated orthotopic position of the stents at the time of implantation and after 4 weeks. During the deployment procedure, rhythm disturbances occurred in all animals. The peak-to-peak transvalvular gradient was $2.3 \pm 1.2 \mathrm{~mm} \mathrm{Hg}$ initially and $4.1 \pm 2.4 \mathrm{~mm} \mathrm{Hg}$ at followup. One-month follow-up confirmed competent neovalves without any paravalvular leakage. Gross morphology demonstrated good opening and closure characteristics. No calcification was seen macroscopically, and surrounding tissue was free of calcification.

Conclusion: In the present study we demonstrated successful merging of 2 novel technologies for percutaneous treatment of pulmonary valve diseases using polyurethane stent valve constructs. (J Thorac Cardiovasc Surg 2010;139:748-52)

Percutaneous pulmonary and aortic valve implantations were clinically introduced by Bonhoeffer and colleagues ${ }^{1}$ in 2000 and Cribier and associates ${ }^{2}$ in 2002 . This appealing new approach to treating valve disease has made remarkable progress. Recently, Bonhoeffer and colleagues ${ }^{1}$ emphasized the potential effect of percutaneous valved stent implantation on right ventricular outflow tract (RVOT) reintervention. This method proved to be a promising additional and complementary approach to a successful surgical program. ${ }^{3}$ Nevertheless, the resulting anatomic spectrum from pulmonary regurgitation after surgical intervention for congenital RVOT disease is broad. With currently available stents, it is not possible to treat all concerned patients. So far, wide or severely calcified and kinked RVOTs are not suitable for percutaneous therapy. New stent designs and operative

From the Departments of Cardiovascular Surgery ${ }^{\mathrm{a}}$ and Pediatric Cardiology, Christian Albrechts University of Kiel, School of Medicine, Kiel, Germany; and the Department of Applied Medical Engineering, ${ }^{b}$ Helmholtz Institute, RWTH Aachen University, Aachen, Germany.

Disclosures: None.

Supported by the German Heart Foundation for Children, Bonn, Germany (12-072007).

Received for publication June 26, 2009; revisions received July 21, 2009; accepted for publication Aug 9, 2009; available ahead of print Oct 12, 2009.

Address for reprints: Georg Lutter, MD, PhD, Department of Cardiovascular Surgery, Christian Albrechts University of Kiel, School of Medicine, Arnold-Heller-Str. 7, 24105 Kiel, Germany (E-mail: lutter@kielheart.uni-kiel.de).

$0022-5223 / \$ 36.00$

Copyright (c) 2010 by The American Association for Thoracic Surgery doi:10.1016/j.jtcvs.2009.08.013 and interventional hybrid approaches are under investigation to overcome these problems. ${ }^{4,5}$ Our group demonstrated the feasibility of totally percutaneous transfemoral pulmonary valve implantation in an ovine model using self-expanding nitinol stents. ${ }^{6}$ Nevertheless, the overall size of the valved stent should be reduced. With younger patients, especially children, smaller outer diameters of the delivery system $(<18 \mathrm{~F})$ should be used. To circumvent size restrictions of percutaneous heart valves, we designed a novel low-profile valved stent made of polyurethane that fits into a $14 \mathrm{~F}$ device. $^{7}$

The aim of this study was to evaluate the functioning of percutaneously implanted self-expanding stents carrying a polyurethane valve in the pulmonary position. This study was performed in juvenile sheep during a 1-month period using angiographic, echocardiographic, hemodynamic, and macroscopic analyses and histologic assessment.

\section{MATERIALS AND METHODS \\ Animal Model and Implantation Procedure}

Nine studies in sheep weighing 32 to $40 \mathrm{~kg}$ are reported. Animals received humane care in accordance with the "Principles of laboratory animal care" and the "Guide for the care and use of laboratory animals" (National Institutes of Health, publication no. 85-23, revised 1985). An ethical committee on animal research at Kiel University approved the protocol.

Anesthesia and implantation were carried out as previously described. ${ }^{6}$ Seven-French sheaths were introduced into the left femoral artery and vein of 7 sheep for hemodynamic measurement. The novel low-profile 


\section{Abbreviations and Acronyms \\ RVOT $=$ right ventricular outflow tract \\ TTE $=$ transthoracic echocardiography}

valved stent was made of polyurethane, which fits into a $14 \mathrm{~F}$ delivery device (Figure 1). This was percutaneously inserted into the right groin, and the valved stents were deployed directly over the native pulmonary valve under fluoroscopic control. ${ }^{6}$ The delivery system was removed, as previously described, ${ }^{6}$ and animals were analyzed for various parameters, as described below. Afterward, the sheep were returned to the animal facility, where their general health was checked daily. They were not treated for anticoagulation or inhibition of platelet aggregation. One month later, the animals were reanalyzed (see below) and killed.

\section{Stent Design}

Radially self-expanding nitinol stents (Nitinol Devices \& Components, Inc, Fremont, Calif) were used in the present study. The stent wires are soft and highly malleable, molding 2 ranks of 14 rhombs each. Bare stents were $28 \mathrm{~mm}$ in length and 22 or $24 \mathrm{~mm}$ in diameter when fully expanded. We used a dip-coating technique. Polyurethane was dissolved with an organic dissolver, and after evaporation of the solvent, the valve was cured.

\section{Angiography}

An angiographic unit (Optimus M 200; Philips Medizin Systeme, Hamburg, Germany) was used for fluoroscopic assessment of the position and function of the polyurethane valves.

\section{Echocardiographic and Hemodynamic Measurements}

Transthoracic echocardiography (TTE) was performed at 4 weeks' follow-up, as previously described. ${ }^{6}$ Arterial pressure, right ventricular pressure, and pulmonary artery pressure were measured, as previously described. ${ }^{6}$

\section{Macroscopic Examination}

Valved stents were grossly inspected, and photographs were taken. Special attention was given to confirm retraction of the cusps or detect any deformed or indurated parts of the valve. The atrial and ventricular chambers and the pulmonary artery were exposed to look for catheter-induced damage or penetration of stent struts.

\section{Histologic Analysis}

The surrounding tissue of the pulmonary artery was fixed in $4 \%$ formalin for 72 hours, soaked in tap water for 2 hours, dehydrated in graded alcohol, and finally embedded in paraffin (Merck, Darmstadt, Germany). Sections (1-2 $\mu \mathrm{m})$ of each tissue sample were deparaffinized and rehydrated. For general morphology, serial sections were stained with hematoxylin and eosin stain (cellular components and nuclei). For identification of calcification, von Kossa staining was conducted. The slides were thoroughly rinsed, with repeated turning of the slide during staining to avoid edge-effect artifacts. Sections were analyzed and documented by using common bright-field light microscopy (Axiovert S 100; Zeiss, Jena, Germany).

\section{Statistics}

Values are presented as means \pm standard deviations. Data were analyzed with the paired Student $t$ test to compare related normally distributed data, and nonnormally distributed data by using SPSS 10.1 software (SPSS, Inc, Chicago, Ill). $P$ values of less than .05 were considered statistically significant.

\section{RESULTS}

Eight of 9 sheep from the study survived the entire 4-week observation period. One sheep died due to ventricular arrhythmia during the implantation procedure, and 1 sheep had endocarditis. Data were analyzed from all other sheep $(n=7)$. The mean diameter of the pulmonary annuli was $17.7 \pm 0.6 \mathrm{~mm}$ (range, 17.0-18.0 mm), as revealed by angiography.

Implanted valved stents with a maximal outer diameter of 22.1 to $24.2 \mathrm{~mm}$ were used. The mean duration of the procedure from insertion of the application device through the sheath to deployment was 56 seconds.

\section{Echocardiography}

TTE demonstrated a laminar blood flow across the valved stents in the analyzed cases. One mild central insufficiency and no paravalvular leakage were detected. Left ventricular and right ventricular function and dimensions appeared normal. The mean systolic internal diameter of the valved nitinol stents was measured at $16.5 \pm 1.5 \mathrm{~mm}$, and the mean internal diastolic diameter was measured at $19.6 \pm 0.7 \mathrm{~mm}$.

\section{Angiography}

Angiography showed orthotopic positioning of the stents at the time of implantation in all animals. In contrast, all animals demonstrated orthotopic position, with the exception of 1 animal, in which the stent was shown to be in the pulmonary artery at approximately $1 \mathrm{~cm}$ distal of the base of the pulmonary valve after 4 weeks. One-month angiography demonstrated good opening and closure characteristics. Angiography revealed competent valved stents in all 7 surviving sheep after 4 weeks (Figure 2). Paravalvular leakages were not observed.

\section{Hemodynamic Measurements}

During the deployment procedure, rhythm disturbances occurred in all animals. The peak-to-peak gradient across the valved stent was $2.3 \pm 1.2 \mathrm{~mm} \mathrm{Hg}$ initially and $4.1 \pm$ $2.4 \mathrm{~mm} \mathrm{Hg}$ at follow-up. Hemodynamic data are shown in Table 1.

\section{Macroscopic Examination}

Postmortem examination after 1 month confirmed the correct position of the valved stent in 6 of 7 sheep (Figure 3, A). In 1 sheep the valved stent was found in the pulmonary artery at approximately $1 \mathrm{~cm}$ distal to the base of the pulmonary valve.

No paravalvular defects were visible. The valved stents were pliable, and the leaflets were thin without indurations. Slight fibrous overgrowth was seen at the outflow portions of the valved stents in 3 of 7 cases (Figure 3,B). Cardiac structures were unscathed. No macroscopic damage was noted. In particular, there was no penetration of any of the stent 

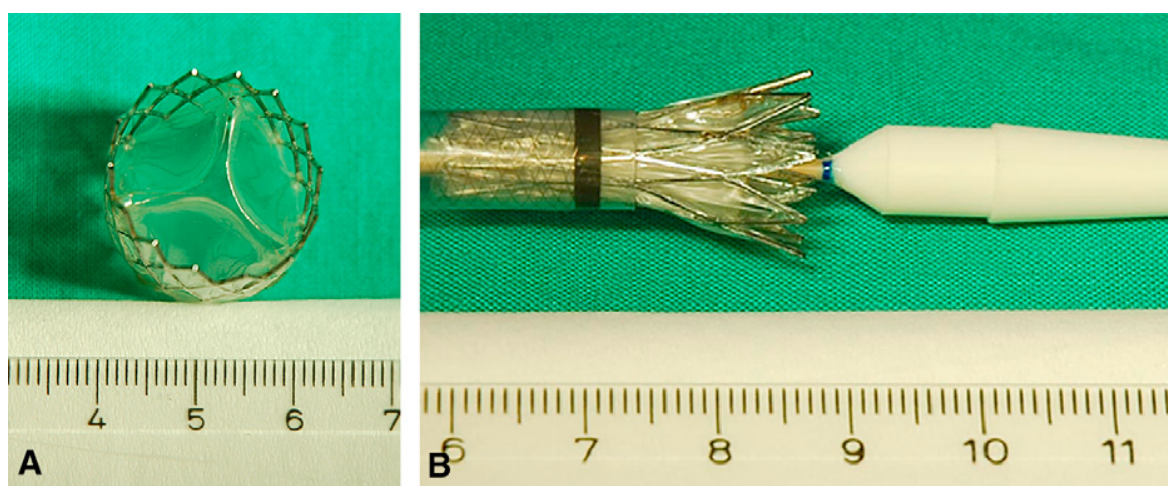

FIGURE 1. A, Top view of a self-expanding nitinol stent (length, $28 \mathrm{~mm}$; diameter, $22 \mathrm{~mm}$; Nitinol Devices \& Components, Inc) containing a 3-leaflet polyurethane valve. B, Lateral view of the top of the delivery system including a polyurethane valved stent.

struts. No inflammation in the surrounding area was visible macroscopically.

\section{Histology}

The surrounding tissue of the pulmonary artery showed normal hematoxylin and eosin staining (Figure 4, $A$ ) and no calcification after von Kossa staining (Figure 4, B).

\section{DISCUSSION}

Surgical reconstruction of the RVOT in children with congenital heart defects, including pulmonary valve replacement, is connected with low morbidity and mortality, ${ }^{8}$ and implantation of a pulmonary homograft is considered the gold standard. However, graft deterioration is very common, and therefore reoperations are often unavoidable in adoles-

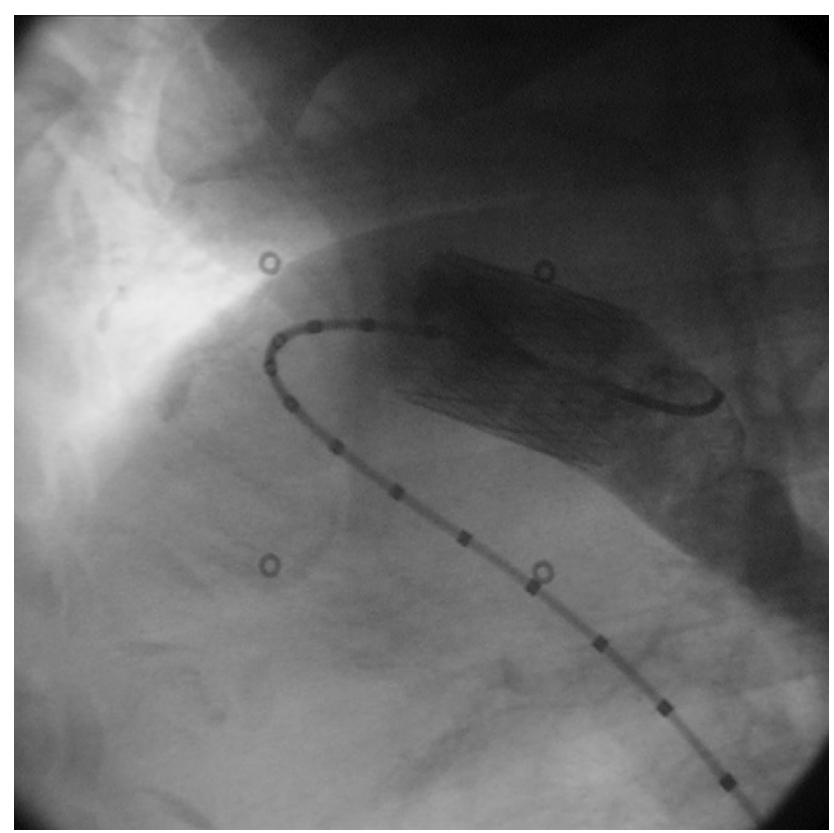

FIGURE 2. Correct position of the polyurethane valved stent in the pulmonary position in an ovine model. After 4 weeks, no regurgitation was assessed. cents. ${ }^{9}$ In contrast, percutaneous pulmonary valve implantation is emerging as an alternative or additional option for a successful surgical scheme, recently being introduced into clinical practice. ${ }^{1}$ Bonhoeffer and colleagues ${ }^{10}$ successfully performed 155 procedures with no early deaths and only 4 procedural complications. The survival rate of these 155 patients after 83 month was $96.9 \%$. The advantages of minimally invasive procedures are obvious (ie, smaller incisions, less postoperative pain, shorter hospital stay, and lower costs). Therefore balloon-expandable valved stents are already used in clinical practice. ${ }^{3}$ The advantage of xenografts is no need of anticoagulation and a high biocompatibility. For homografts, there is a relative resistance against infections and a high biocompatibility. The disadvantage of both is that they have no growth potential and a limitation of durability and degeneration. Transcatheter valves were implanted under fluoroscopic control, and this implantation procedure is better for the patient.

The potential advantages of nitinol stents regarding preservation of the valve and deployment have been previously discussed. ${ }^{6}$

In this study we used the percutaneous polyurethane valved stent implant, which fits into a $14 \mathrm{~F}$ delivery device. ${ }^{7}$ Polyurethane has demonstrated the best biocompatibility, durability, and resistance to thromboembolism among all polymers, and therefore it was also used for a variety of medical devices. However, until now, even though manufactured polyurethane has had sufficient mechanical strength and flexibility for heart

TABLE 1. Hemodynamic data

\begin{tabular}{lcccc}
\hline & \multicolumn{4}{c}{ Valved stent implantation } \\
\cline { 2 - 5 } & Before & After & 1-mo follow-up & $\boldsymbol{P}$ value \\
\hline RVP, systolic & $15 \pm 0$ & $15.5 \pm 0$ & $20.3 \pm 6.5$ & $<.0006$ \\
RVP, diastolic & $4 \pm 0$ & $4 \pm 0.5$ & $2.4 \pm 1.9$ & $\mathrm{NS}$ \\
RVP, mean & $9 \pm 0$ & $8.5 \pm 0.25$ & $8.8 \pm 3.3$ & $\mathrm{NS}$ \\
PAP, systolic & $12.3 \pm 0.8$ & $12.5 \pm 0.9$ & $18 \pm 3.4$ & $<.001$ \\
PAP, diastolic & $9 \pm 0.6$ & $9.5 \pm 0.8$ & $6.2 \pm 3.8$ & $\mathrm{NS}$ \\
PAP, mean & $10.1 \pm 0.4$ & $10.5 \pm 0.6$ & $12 \pm 2.9$ & $\mathrm{NS}$ \\
\hline
\end{tabular}

$R V P$, Right ventricular pressure; $P A P$, pulmonary artery pressure. 

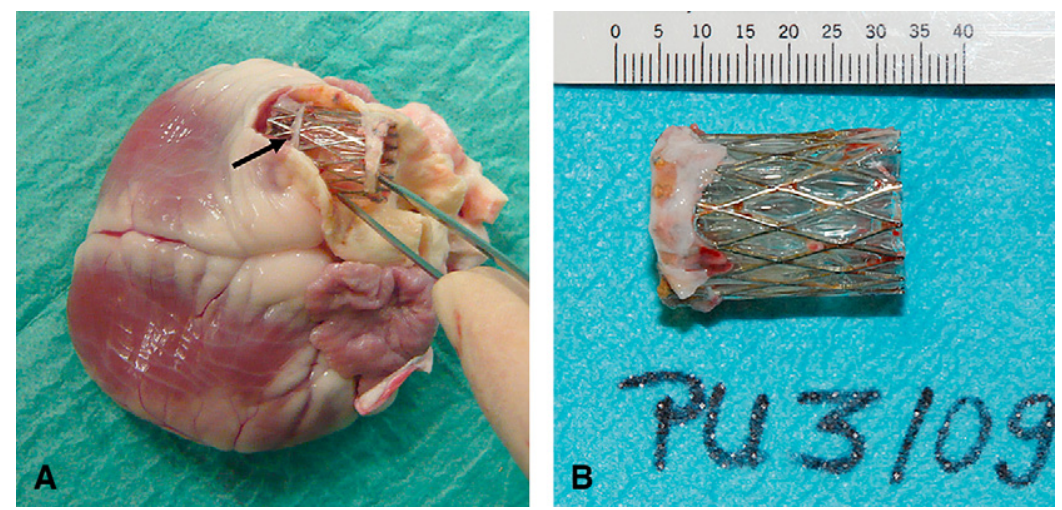

FIGURE 3. Gross morphology of polyurethane pulmonary valved stent. A, The native pulmonary valve can be seen (arrow). B, At the distal part of the stent, mild fibrosis was observed at the explanted stent.

valve leaflets with acceptable long-term fatigue properties in vitro, it has performed disappointingly when implanted in vivo. Polyurethane is one of the most important categories of synthetic polymers used for both short- and long-term blood-contact medical applications. The development of polymeric heart valve substitutes goes back to the end of the 1950s, when Roe and Moore ${ }^{11}$ implanted an aortic prosthesis made of silicone rubber in human subjects. Many polymeric heart valves were tested in vitro and in vivo, including valves made of silicone, polytetrafluoroethylene (Teflon; Mecora, Aachen, Germany), polyethylene terephthalate (Dacron; Mecora), polyvinylchloride, and polyurethane. None of these valves proved to be adequate for human implantation because material degradation and thrombogenicity remained unsolved problems. In the 1980s and 1990s, new insight was obtained to show that durability not only depends on the valve polymer but also is mainly influenced by the manufacturing process and design. The aim was to manufacture a so-called biomechanical valve, a prosthesis that is made only of artificial material. Nevertheless, the valve is flexible because of the material properties of polyurethane. Now the hope is to combine 2 advantages: long-term durability and no necessity for permanent anticoagulation.

In the present study angiographic examination mainly demonstrated correct positioning and a good adjustment of the pulmonary diameter, annular size, and valved stent diameter. The soft nitinol stent's ability to adapt to the surrounding structure was conducive to physiologic blood flow in the great arteries: the new valved stent did not negatively affect right or left ventricular function, which was shown by hemodynamic measurements. The echocardiogram demonstrated the same contractility and relaxation properties of the left and right ventricles before and after implantation of the polyurethane valve. A low transvalvular gradient was seen before implantation and after 1 month follow-up. Macroscopic and histologic assessment revealed no calcifications.

In this study we confirmed the implantability of our new polyurethane valved stent. During the 4-week observation period, no defects of the leaflets were detected. Angiography demonstrated orthotopic position and showed good opening and closing capacity. Neither immune reactions nor thrombosis was observed. In contrast, mild fibrosis was seen in the distal part of the valved stent. Therefore it was not necessary to use any anticoagulation or immune-suppressive medication.

Wheatley and coworkers ${ }^{12}$ used polyurethane, bileaflet mechanical (ATS Medical, Inc, Minneapolis, Minn), and Carpentier-Edwards porcine (Edwards Lifesciences, Irvine, Calif) valves for their study. These valves were implanted in the mitral position of growing sheep and explanted after 6
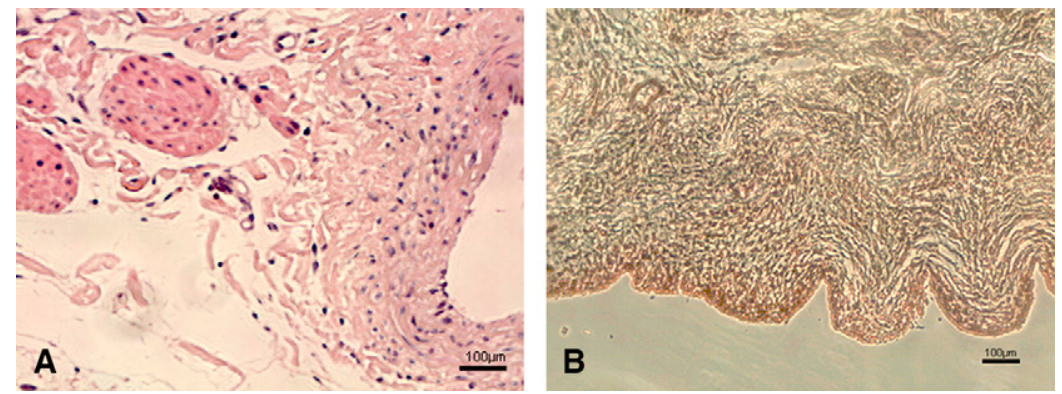

FIGURE 4. Histologic analyses. A, Normal structure and cells of the surrounding tissue were seen with the overview by means of hematoxylin and eosin staining. B, Von Kossa staining of the surrounding tissue was conducted for calcification. No calcifications were detected. 
months, and no anticoagulation medication was used. They showed that the thrombogenicity of the polyurethane valves was lower compared with that of the mechanical valves. In addition, this research group indicated that the polyurethane valve in the mitral position was superior to a bioprosthesis.

Daebritz and associates ${ }^{13}$ analyzed the implantation of a flexible, polymeric aortic valve, which underwent longterm in vitro and in vivo testing in a growing calve animal model. These polyurethane aortic heart valves showed excellent in vitro and in vivo hemodynamics after 20 weeks. They were able to show that surgically implanted, flexible, polymeric heart valve prosthesis was superior to current bioprosthesis in animal testing. The same type of polyurethane valves has been used in our valved stent study. The thickness of the polyurethane membrane is 100 to $150 \mu \mathrm{m}$, and the thickness of the bovine jugular vein is $230 \pm 50 \mu \mathrm{m}^{14}$

Furthermore, Hashimoto and colleagues ${ }^{15}$ used an umbrella-shaped polyurethane valve, which was attached to the tip of newly devised repositionable stent valve in the aortic position. They analyzed 2 different groups, one with polyurethane valves and the second with the stentless valve. In their study aortographic analysis of the first group showed good function, and the diastolic pressure indicated no significant change. The other group (without the valve) showed massive aortic regurgitation followed by dysfunction of the native valve. These experiments were done in pigs, and they were killed 1 hour after implantation. The postmortem examination showed small amounts of thrombus inside the polyurethane umbrella in group 1 .

In this study we have demonstrated an excellent outcome of the polyurethane valves in the pulmonary position in a sheep model after 4 weeks. The results of our study showed that the polyurethane valve can be used as a new stented heart valve in a period of 4 weeks.

\section{CONCLUSION}

Percutaneously implanted polyurethane valves were evaluated during a 1-month period in an ovine model. The sheep hearts demonstrated good structural and functional outcome.
We thank Christine Haß and Marion Krüger for their excellent technical assistance and Klaus Mück for preparing the application devices.

\section{References}

1. Bonhoeffer P, Boudjemline Y, Saliba Z, Merckx J, Aggoun Y, Bonnet D, et al. Percutaneous replacement of pulmonary valve in a right-ventricle to pulmonary-artery prosthetic conduit with valve dysfunction. Lancet. 2000;356:1403-5.

2. Cribier A, Eltchaninoff H, Bash A, Borenstein N, Tron C, Bauer F, et al. Percutaneous transcatheter implantation of an aortic valve prosthesis for calcific aortic stenosis: first human case description. Circulation. 2002;106:3006-8.

3. Coats L, Tsang V, Khambadkone S, vann Doorn C, Cullen S, Deanfield J, et al. The potential impact of percutaneous pulmonary valve stent implantation on right ventricular outflow tract re-intervention. Eur J Cardiothorac Surg. 2005;27: 536-43.

4. Zhou JQ, Corno AF, Huber CH, Tozzi P, von Segesser LK. Self-expandable valved stent of large size: off-bypass implantation in pulmonary position. Eur $J$ Cardiothorac Surg. 2003;24:212-6.

5. Boudjemline Y, Schievano S, Bonnet C, Coats L, Agnoletti G, Khambadkone S, et al. Off-pump replacement of the pulmonary valve in large right ventricular outflow tracts: a hybrid approach. J Thorac Cardiovasc Surg. 2005;129:831-7.

6. Attman T, Quaden R, Jahnke T, Muller-Hulsbeck S, Boening A, Cremer J, et al. Percutaneous pulmonary valve replacement: 3-month evaluation of self-expanding valved stents. Ann Thorac Surg. 2006;82:708-14.

7. Attmann T, Steinseifer U, Cremer J, Lutter G. Percutaneous valve replacement: a novel low-profile polyurethane valved stent. Eur J Cardiothorac Surg. 2006; $30: 379$.

8. Kanter KR, Budde JM, Parks WJ, Tam VK, Sharma S, Williams WH, et al. One hundred pulmonary valve replacements in children after relief of right ventricular outflow tract obstruction. Ann Thorac Surg. 2002;73:1801-6.

9. Wells WJ, Arroyo H Jr, Bremner RM, Wood J, Starnes VA. Homograft conduit failure in infants is not due to somatic outgrowth. $J$ Thorac Cardiovasc Surg. 2002; 124:88-96.

10. Lurz P, Coats L, Khambadkone S, Nordmeyer S, Boudjemline Y, Schievano S, et al. Percutaneous pulmonary valve implantation impact of evolving technology and learning curve on clinical outcome. Circulation. 2008;117:1964-72.

11. Roe BB, Moore D. Design and fabrication of prosthetic valves. Exp Med Surg. 1958; 16:167-77.

12. Wheatley DJ, Raco L, Bernacca GM, Sim I, Blecher PR, Boyd JS. Polyurethane: material for the next generation of heart valve prosthesis? Eur J Cardiothorac Surg. 2000;17:440-8.

13. Daebritz SH, Fausten B, Hermanns B, Schroeder J, Groetzner J, Autschbach R, et al. Introduction of a flexible polymeric heart valve prosthesis with special design for aortic positioning. Eur J Cardiothorac Surg. 2004;25:946-52.

14. Li W, Liu WY, Yi DH, Yu SQ, Jin ZX. Histological/biological characterization of decellularized bovine jugular vein. Asian Cardiovasc Thorac Ann. 2007;15:91-6.

15. Hashimoto M, Kaminou T, Ohuchi Y, Nakamura K, Sugiura K, Dachi A, et al. Development of a re-positionable aortic stent-valve: a preliminary study in swine. J Interv Cardiol. 2008;21:432-40. 